\title{
In vivo visualization of uterine mast cells by two-photon microscopy
}

\author{
Franziska Schmerse, Katja Woidacki, Monika Riek-Burchardt ${ }^{1,2}$, Peter Reichardt ${ }^{2}$, Axel Roers ${ }^{3}$, \\ Carlos Tadokoro ${ }^{4}$ and Ana Claudia Zenclussen \\ Experimental Obstetrics and Gynecology, Medical Faculty, Otto-von-Guericke University Magdeburg, Gerhart- \\ Hauptmann-Street 35, 39108 Magdeburg, Germany, ${ }^{1}$ Project Group Neuropharmacology, Leibniz Institute for \\ Neurobiology, 39118 Magdeburg, Germany, ${ }^{2}$ Institute for Molecular and Clinical Immunology, Otto-von-Guericke \\ University Magdeburg, 39120 Magdeburg, Germany, ${ }^{3}$ Medical Faculty 'Carl Gustav Carus', Institute of Immunology, \\ 01307 Dresden, Germany and ${ }^{4}$ Instituto Gulbenkian de Ciências, Oeiras, Portugal
}

Correspondence should be addressed to A C Zenclussen; Email: ana.zenclussen@med.ovgu.de KWoidacki; Email: katja.woidacki@med.ovgu.de

\begin{abstract}
Transgenic mice expressing fluorescent proteins in specific cell populations are widely used for the study of in vivo behavior of these cells. We have recently reported that uterine mast cells (uMCs) are important for implantation and placentation. However, their in vivo localization in uterus before and during pregnancy is unknown. Herein, we report the direct observation of uMCs in vivo using doubletransgenic C57BL/6J Mcpt5-Cre ROSA26-EYFP mice with high expression of enhanced yellow fluorescent protein in MC protease 5 (Cma1 (Mcpt5))-expressing cells by intravital two-photon microscopy. We were able to monitor MCs live in utero during the murine estrous cycle and at different days of pregnancy. We demonstrated that uMCs accumulated during the receptive phase of the female (estrus) and persisted in large numbers at early pregnancy stages and around mid-gestation and declined in number in non-pregnant animals at diestrus. This intravital microscopy technique, including a custom-made microscope stage and the adaption of the surgical procedure, allowed the access of the uterus and implantations for imaging. The introduced application of intravital microscopy to C57BL/6J-Mcpt5-Cre ROSA26-EYFP mice offers a novel and powerful in vivo approach to further address the evident relevance of uMCs to reproductive processes with obvious clinical implications.

Reproduction (2014) 147 781-788
\end{abstract}

\section{Introduction}

Besides the well-documented role of mast cells (MCs) in IgE-mediated allergic responses (Galli \& Tsai 2012), MCs appear now as key effector cells influencing a variety of physiological as well as pathophysiological processes (Bischoff 2009, Anand et al. 2012). Not only do they contribute to innate and adaptive immune responses (Galli et al. 2005, St John \& Abraham 2013), but also they are directly involved in non-immunological processes including tissue remodeling and angiogenesis (Varayoud et al. 2004, Bosquiazzo et al. 2007, Theoharides et al. 2010). Their effects are mainly mediated by granule-stored mediators such as metalloproteases, MC-specific proteases, tissue plasminogen activator (tPA), vascular endothelial growth factor (VEGF), transforming growth factor $\beta$ (TGF $\beta$ ) (Galli et al. 2005) and galectin 1 (Gal1, Woidacki et al. 2013) that are released upon activation. We have recently reported that MCs migrate from the periphery to the uterus responding to hormonal fluctuations
(Jensen et al. 2010). This has already suggested their importance for pregnancy, a period characterized by great hormonal changes. In a mouse model, Kit (c-Kit) deficiency, which is associated with a lack of MCs including uterine MCs (uMCs), was related to impaired implantation and placentation that negatively influenced fetal growth (Woidacki et al. 2013). We found these effects to be mediated by Gal1 as bone marrow-derived MCs (BMMCs) from WT mice but not from Lgals1 (Gal1)deficient mice could rescue the impaired reproductive phenotype (Woidacki et al. 2013). The in vivo behavior of MCs at the fetal-maternal interface is, however, largely unknown. As there were some controversial reports on the role of MCs in pregnancy (Salamonsen et al. 1996, Varayoud et al. 2004, Menzies et al. 2012, Woidacki et al. 2013), it is of vital importance to localize uMCs at the fetal-maternal interface and to later dissect their behavior and interaction with other cells live.

Transgenically modified mice with fluorescentlabeled MCs using MC-specific Cre recombinase/ loxP-mediated recombination are very useful for the 
visualization of MCs in vitro but particularly in vivo (Luche et al. 2007, Scholten et al. 2008). In 2001, the production of Cre reporter alleles that express enhanced yellow fluorescent protein (EYFP), a color variant of the green fluorescent protein, was described (Srinivas et al. 2001). In this mouse model, EYFP was inserted into the ubiquitously expressed ROSA26 locus, framed by a loxPflanked stop sequence. Crossing these ROSA26-EYFP strains with transgenic strains expressing Cre either in a ubiquitous or in a cell-specific pattern would now allow monitoring Cre activity in living tissues (Srinivas et al. 2001). Scholten et al. (2008) were the first to describe the combination of the Mcpt5-Cre transgenic mouse line crossed with the ROSA26-EYFP Cre excision reporter strain. Due to the lack of Mcpt5-Cre transgene expression in MCs of the mucosal type, EYFP signal was detected exclusively in connective tissue-type MCs (Dudeck et al. 2011) and the serine protease Mcpt5 showed unique tissue specificity for connective tissuelike MCs (Lützelschwab et al. 1998).

Two types of MCs are known: mucosal and connective tissue-type MCs. This classification is based on phenotypic differences rather than on their tissue localization (Metcalfe et al. 1997, Schwartz \& Huff 1998). Additionally, for rodents, a third population is described. These cells show characteristics of both mucosal and connective tissue-type MCs (Schwartz \& Huff 1998, Michaloudi \& Papadopoulos 1999, Woidacki et al. 2013). Unfortunately, to the best of our knowledge, no model is currently available in which both types of MCs can be visualized in vivo. Although the percentage of Mcpt5-expressing MCs from isolated uterine cells ranged between 5 and 20\% (Woidacki et al. 2013), double-transgenic C57BL/6)Mcpt5-Cre ROSA26-EYFP mice with high expression of EYFP in Mcpt5-expressing cells emerge as a powerful tool for the visualization of uMCs live and in vivo by twophoton microscopy. Here, the simultaneous absorption of two photons, which precedes the emission of the fluorescence, is harmless to the tissue, avoids photobleaching and enables a better tissue penetration (Paoli et al. 2009). Based on these methodological advantages, two-photon microscopy became a preferred technique for intravital imaging (Zipfel et al. 2003, Olivieri \& Tadokoro 2013). Until recently, the visualization of cells in vivo at the feto-maternal interface was hampered by the fact that in vivo microscopy was not established for the uterus or the placenta. Recently, we have succeeded in visualizing the feto-maternal interface in vivo by modifying the technique for intravital imaging (Zenclussen et al. 2012, 2013).

In this study, the application of this intravital technique to C57BL/6J-Mcpt5-Cre ROSA26-EYFP mice provided direct observations of $M C$ presence and localization within the virgin and pregnant mouse uteri live and in vivo. This information opens up vast new possibilities to understand the behavior and functional relevance of uMCs in health and disease.

\section{Materials and methods}

\section{Animals}

Mcpt5-Cre mice (Scholten et al. 2008) were crossed with R26 StopFLOX-EYFP reporter mice (Srinivas et al. 2001). Cre reporter strains were produced by targeted insertion of EYFP and ECFP into the ROSA26 locus, which express EYFP in cells with a deleted loxP-flanked stop element. These mice were then housed at the Central Animal Facility at the Otto-vonGuericke University Magdeburg, Medical Faculty, with a $12 \mathrm{~h}$ light:12 h darkness cycle. In all experiments, mice at the age of 10-16 weeks were used to avoid age-dependent influences on their reproductive phenotype (Talbert 1971, Parkening et al. 1978, Holinka et al. 1979). All procedures were in accordance with the institutional guidelines of Magdeburg, and the study was previously approved by the Landesdirektion Dessau (42502-2-978 Magdeburg). This is in agreement with the Guide for the Care and Use of Agricultural Animals in Agricultural Research and Teaching, USA.

A vaginal lavage was performed to determine the exact state of the estrous cycle by analyzing the content and type of cells in the vaginal smear. For this, the vagina was gently washed with $20 \mu \mathrm{l}$ 0.9\% sodium chloride solution (Berlin-Chemie, Berlin, Germany). For identification of the state of the estrous cycle, the cells were distributed on an object slide and analyzed by light microscopy with a total magnification of $200 \times$ (ECLIPSE-S, Nikon, Düsseldorf, Germany). For in vivo imaging of the virgin uterus, we focused on diestrus and estrus as we previously found these two phases to be the time points with major differences in the number of MCs between each other (Woidacki et al. 2013). For in vivo imaging of the pregnant uterus, C57BL/6J-Mcpt5-Cre ROSA26-EYFP mice were mated allogeneically with BALB/c males. The day of vaginal plug was set as day 0 of pregnancy. Pregnant females were separated from the males upon plug detection. In vivo imaging of the uterus was performed on gestational days $0,2,5,8,10$ and 14 .

\section{Processing of the uterus/implantations for the two-photon microscopy technique}

Animals were anesthetized by s.c. injection of ketamine (CPPharma, Burgdorf, Germany) (125 $\mu \mathrm{g} / \mathrm{g}$ body weight of mouse) and Rompun (Bayer Health Care, Bayer Vital GmbH) $(50 \mu \mathrm{g} / \mathrm{g}$ body weight of mouse). After s.c. anesthetization, mice were ventilated through a tracheal tube with a mechanical small animal respirator (Mini-Vent, Hugo Sachs Elektronik, MarchHugstetten, Germany) receiving a mixture of 0.6 vol\% isoflurane (Baxter, Unterschleißheim, Germany) and oxygen for narcosis using a Univentor 400 Anaesthesia Unit (Univentor, Zejtun, Malta).

Animals were kept on top of a heating pad at $37^{\circ} \mathrm{C}$ and hairs at the abdomen were removed using depilatory cream (Veet, Mannheim, Germany). Fluorescence labeling of blood vessels was done by retro-orbital injection of $100 \mu$ l Rhodamine B (10 mg/ml, Sigma-Aldrich) or $12.5 \mu \mathrm{l}$ QTracker 655 nontargeted QDots (Invitrogen) dissolved in $80 \mu$ aqua ad injectabilia (Berlin-Chemie). The uterus could be accessed by a lateral section at the abdomen. The female mouse and its uterine horns were stabilized on a microscope stage (custom-made design, 
Hasomed, Magdeburg, Germany). One of the uterine horns was carefully exposed and fixed using an aluminum foil fixator (self-made), which was placed under the uterine horn without disrupting it. Uterine contractions were reduced by drenching the organ with caffeine $(20 \mathrm{mg} / \mathrm{ml}$, Sigma-Aldrich Chemie $\mathrm{GmbH}$ ). Caffeine was provided continuously in dissolved form using caffeine-soaked cotton. We have shown previously that the addition of caffeine can prevent uterine muscle contraction without any effect on blood circulation (Zenclussen et al. 2013). Low-melting agarose (2\% in PBS, Peqlab, Erlangen, Germany) was carefully placed around and above the whole uterus to keep the organ at physiological temperatures. After preparation, a heater with a glass-covered opening was placed on top. The temperature probe connected to this heater was placed in very close proximity to the organ where a temperature of $37^{\circ} \mathrm{C}$ was maintained throughout the experiment.

\section{Imaging of uMCs by two-photon microscopy}

For imaging of the uterus, two-photon microscopy was performed using a Zeiss LSM-710 microscope (Carl Zeiss, Jena, Germany) with simultaneous detection via three external non-descanned detectors. The microscope was equipped with a MaiTai TiSa laser (Newport/Spectra-Physics, Darmstadt, Germany) on an AxioExaminer upright stage and a waterdipping lens (NA 1.0, magnification $\times 20$ ). Illumination was performed at a wavelength of 850 or $950 \mathrm{~nm}$. EYFP-positive MCs were detected at 500-550 nm emission. Blood vessels were labeled either with Rhodamine B and detected with a bandpass filter of 565-610 nm or with QTracker 655 nontargeted QDots detected at 640-710 nm. Tissue structure was detected by its second-harmonic generation ( $\mathrm{SHG}$ ) signal below $480 \mathrm{~nm}$ based on the non-centrosymmetric structure of collagen (Williams et al. 2005). Images were recorded as z-stacks overarching the whole uterine horn with $3 \mu \mathrm{m}$ Z-spacing using Zen software 2009 (Carl Zeiss). For 3D reconstruction, Volocity software (Perkin Elmer/Improvision, Waltham, MA, USA) was used. Images are representative for three observations in total.

\section{Quantification of uMCs}

The number of MCs was determined in representative z-stacks of uteri from virgin females either at diestrus or estrus of the estrous cycle ( $n=5$ /each) or during pregnancy (days $0,2,5,8,10$ and 14) $(n=3$ per stage) using Zeiss LSM Image Browser software (Carl Zeiss Microlmaging $\mathrm{GmbH}$ ). The mean number of MCs was calculated as the total number of MCs per recorded z-layer of one z-stack. Statistical analysis was performed by ANOVA test, followed by post hoc Tukey's multiple comparison test.

\section{Results}

\section{Two-photon microscopy represents a powerful tool for} the visualization of uMCs live and in vivo

We first visualize uMCs intravitally in surgically exposed virgin and pregnant murine uteri. Application of twophoton laser scanning microscopy allowed the live imaging of uMCs up to depths of $\sim 200 \mu \mathrm{m}$ in double- transgenic C57BL/6J-Mcpt5-Cre ROSA26-EYFP mice, depending on biological divergences such as pregnancy stage and technical implementation. Compared with the methods applied to fixed tissue, the intravital microscopy technique allowed us to detect uMCs live.

Caffeine was used for reducing the intensive uterine contractions at a concentration of $20 \mathrm{mg} / \mathrm{ml}$. Its application allowed high-quality recording of images, 3D renderings and videos. However, day 14 of pregnancy represented the maximum limit for imaging because at later pregnancy stages not only the uterine contractions but also fetal movement are present, and it becomes impossible to reduce fetal and uterine movements without a surgical manipulation of the uterus.

For imaging of the highly blood-perfused, sensitive and contracting uterus, tissue immobilization was evaluated by a new surgical technique. This was performed in accordance with the procedures described previously (Zenclussen et al. 2013). One horn of the murine uterus bicornis was carefully exposed (Fig. 1A), fixed by a self-made aluminum foil fixator and permanently embedded in caffeine-soaked cotton rolls (Fig. 1B) to reduce uterine contractions. As MCs are mainly located in the uterus rather than in placental tissue (Woidacki K, Zenclussen AC, 2010; unpublished observations), we avoided damage to the uterine wall, a procedure shown to be beneficial for imaging of the placenta (Zenclussen et al. 2012). Therefore, the focus was on the mesometrial side of the intact uterus (Fig. 1C) and one implantation (Fig. 1D), with special emphasis on the zone between the uterus and the decidua basalis (Fig. 1D). The permanent moistening of uterine tissue was assured by the addition of low-melting agarose. The implantation to be analyzed was covered by the heating plate containing an opening that allowed the access to the zone of interest (Fig. 1E).

Monitoring of vitality after surgery and during intravital imaging was assured by visualization of the blood flow. In our first experiments, different laser wavelengths were tested to excite simultaneously both EYFP and blood flow. In further preliminary works, Rhodamine B, a red fluorescent blood dye, was used. Implementation of both criteria - simultaneous excitation of EYFP and Rhodamine B at the maximum with one certain wavelength and usage of higher and therefore more tissue-gentle and harmless wavelength revealed that Rhodamine B was not optimal for our setup (data not shown). Therefore, QTracker 655 nontargeted QDots were administrated by retro-orbital injection which allowed the visualization of both blood flow and MCs during the whole imaging process using deep red filter with an absorption wavelength between 640 and $760 \mathrm{~nm}$ for detection of emission of QDots. Visualization of uMCs, blood flow and SHG signal at once was then facilitated by excitation at a wavelength of $950 \mathrm{~nm}$. These fine-modulated settings allowed us to monitor MCs live and in vivo within the virgin as well as 


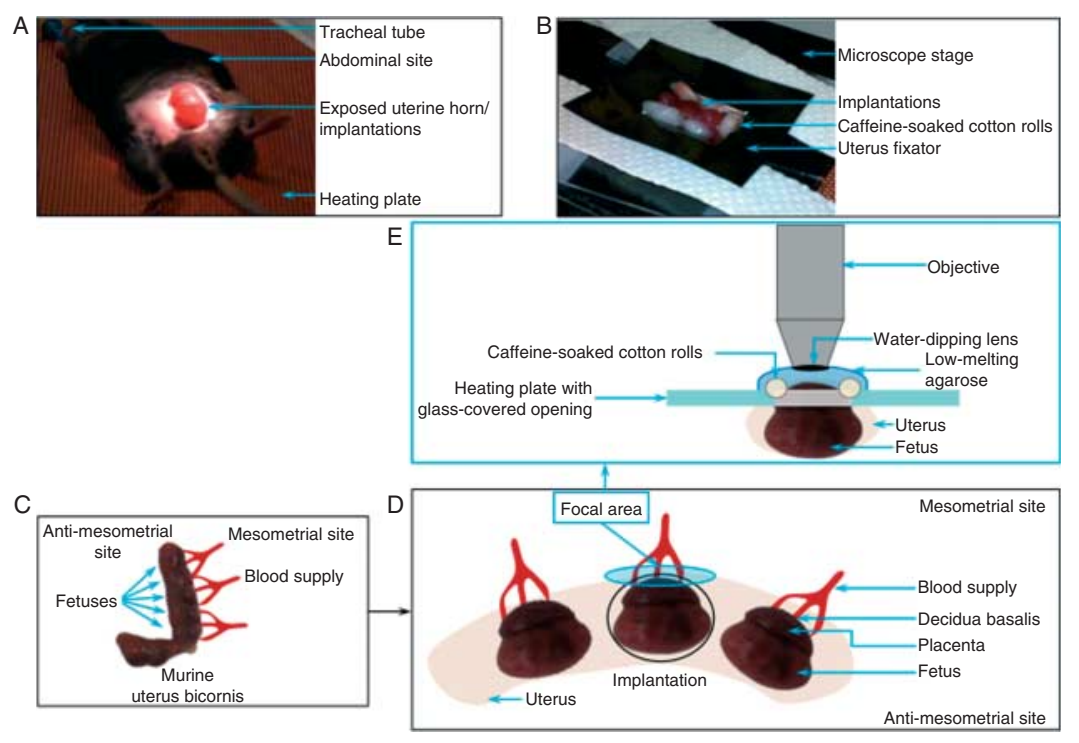

Figure 1 Surgical procedure to make the uterus accessible for applying intravital microscopy. (A) The anesthetized mouse was placed dorsally on a heating plate. One uterine horn of the murine uterus bicornis was exposed surgically to make the implantation accessible for imaging. (B) The basic fixation of the uterus and the implantations respectively was ensured by a custom-made microscope stage. After the fine fixation by a self-made fixator, the uterus/implantations were permanently drained by applying cotton rolls that were soaked with caffeine to reduce uterine contractions during the imaging process. (C) The focal area was on the mesometrial site, more precisely (D) on that zone at which the fetus is in tight contact with the maternal uterine tissue. (E) The implantation was covered by a heating plate containing an opening that allowed the access to the zone of interest.

pregnant uteri of C57BL/6J-Mcpt5-Cre ROSA26-EYFP mice in which Mcpt-5-expressing MCs are positive for EYFP. This is the first report not only in visualizing the pregnant decidua live at different days but also in reporting the presence of MCs in the uterus of a live mouse, confirming the presence of these cells at the fetal-maternal interface.

\section{MCs are present in the virgin uterus and accumulate during the receptive phase of the estrous cycle, the estrus}

By using intravital microscopy together with two-photon microscopy, we could confirm in vivo that uMCs are mainly located in close proximity to blood vessels (Figs 2A, B and 3A, B, C, D, E and F). Furthermore, this method allowed the assessment of the quantitative distribution of uMCs along the murine estrous cycle (Fig. 2A and B). By using flow cytometry and histology, we have recently reported that the number of MCs peaked during the receptive phase of the female, namely at estrus when the uterus is prepared for nidation, while the lowest numbers were observed during diestrus (Woidacki et al. 2013). By using intravital microscopy for detection of EYFP-positive uMCs in C57BL/6J-Mcpt5Cre ROSA26-EYFP mice, we could not only confirm that uMCs accumulate during the receptive phase of the female (estrus, Figs 2A and 4) and their number decline at diestrus (Figs 2B and 4), but also show their density and localization. In previous studies, we could detect uMCs in longitudinally cut uterine sections distributed inter-implantational and in close proximity to the developing fetus (Woidacki et al. 2013). In this study, we observed that uMCs were additionally distributed in large numbers at the mesometrial site of the uterus that is in close proximity to the decidua basalis (Fig. 1D).

\section{uMCs are abundant during early pregnancy and their frequency declines as gestation advances}

To analyze the presence, distribution and density of uMCs during pregnancy, we concentrated first on days 0,2 and 5 of the pre- and peri-implantation phase and later on days 8, 10 and 14 of the post-implantation phase. Uterine tissues at all analyzed pregnancy stages are characterized by remarkably large numbers of Mcpt-5expressing MCs (Figs 3A, B, C, D, E, F and 4). During the pre-implantation phase (days 0 and 2) when the uterus is preparing for the nidation of the blastocyst (Wang \& Dey 2006), an accumulation of uMCs at the mesometrial uterine site was evident (Figs 3A, B and 4). During the implantation process at gestational day 5 , when the blastocyst is in close physical and physiological contact
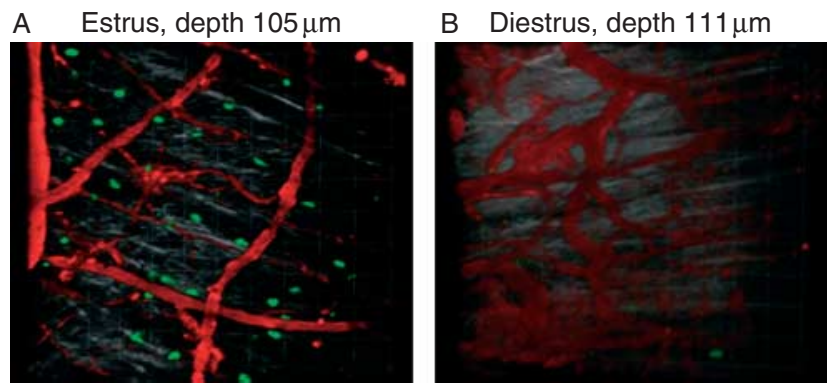

Figure 2 Uterine MCs (uMCs) accumulate at the receptive phase of the female - visualized live by applying intravital two-photon microscopy to $\mathrm{C} 57 \mathrm{BL} / 6 \mathrm{~J}-\mathrm{Mcpt5}$-Cre ROSA26-EYFP mice. UMCs were monitored in virgin C57BL/6J-Mcpt5-Cre ROSA26-EYFP females at two phases of the estrous cycle, namely at estrus (A), which represents the receptive phase of the female and at diestrus (B) and EYFP signal was detected by emission at a wavelength of 500-550 nm. Blood flow was visualized by i.v. injection of QTracker 655 nontargeted QDots and detected by emission at a wavelength of $640-710 \mathrm{~nm}$. Collagen fibers (gray) were detected by its second-harmonic generation (SHG) signal below $480 \mathrm{~nm}$. Images were constructed by 3D rendering of z-stacks. 


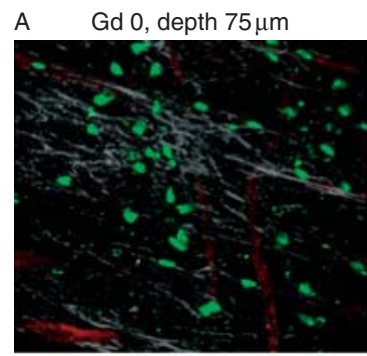

D
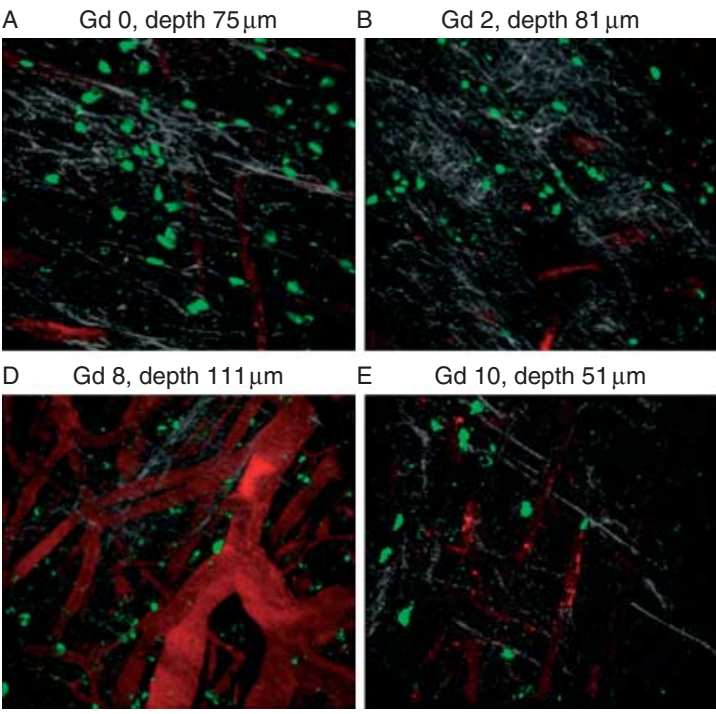

C Gd 5, depth $126 \mu \mathrm{m}$

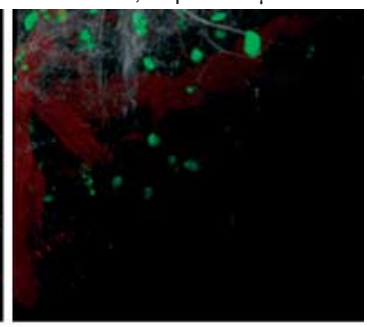

Gd 10, depth $51 \mu \mathrm{m}$

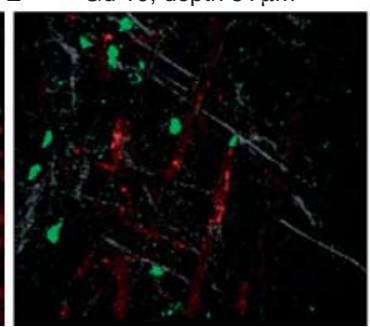

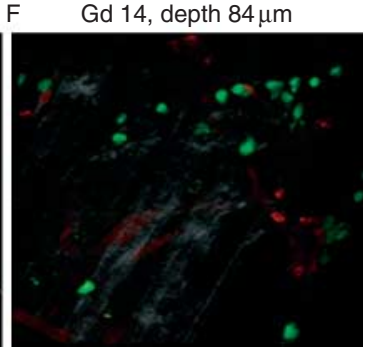

Figure 3 Uterine MCs were monitored in allogenically, BALB/c mated C57BL/6J-Mcpt5Cre ROSA26-EYFP females at gestational days 0 (A, depth $75 \mu \mathrm{m}), 2$ (B, depth $81 \mu \mathrm{m}), 5$ (C, depth $126 \mu \mathrm{m}), 8$ (D, depth $111 \mu \mathrm{m}), 10$ (E, depth $51 \mu \mathrm{m})$ and $14(\mathrm{~F}$, depth $84 \mu \mathrm{m})$ and EYFP signal was detected by emission at $500-550 \mathrm{~nm}$. Blood flow was visualized by i.v. injection of QTracker 655 nontargeted QDots and detected by emission at $640-710 \mathrm{~nm}$. Collagen fibers (gray) were detected by its second-harmonic generation (SHG) signal below $480 \mathrm{~nm}$. to the uterine endometrium (Dey et al. 2004), uMCs were present in comparable numbers as in non-pregnant uteri at estrus albeit this did not reach significance levels when compared with diestrus (Figs $3 \mathrm{C}$ and 4). Around day 8 of gestation when placentation starts (Rugh 1990) and spiral arteries appear histologically within the decidua basalis (Burke et al. 2010), uMCs are present (Fig. 3D) in slightly lower numbers than at early pregnancy (Fig. 4). At gestational day 10 when the placenta and the fetus are simultaneously growing, uMCs could be still detected mesometrially but their number was significantly lower than at estrus (Figs 3E and 4). At mid-gestation (day 14 of pregnancy) when the main focus is on fetal rather than on placental development (Suzuki et al. 1997), uMCs are present but in significantly lower numbers when compared with estrus (Figs 3F and 4).

\section{Discussion}

The in vivo adaptation of two-photon microscopy for monitoring $\mathrm{MC}$ distribution at the fetal-maternal interface offers a novel, impressive and powerful approach to address the evident relevance of uMCs to reproductive processes. The application of two-photon microscopy to transgenic mouse models increases exponentially (Zipfel et al. 2003). Of interest for the reproduction field, it has been recently reported that this methodology can be used for the tracking of dendritic cells (DCs; Zenclussen et al. 2013) and regulatory T cells (Treg; Teles et al. 2013) as well as for the imaging of the mouse placenta under physiological (Zenclussen et al. 2012) as well as pathophysiological conditions (De Moraes et al. 2013).

The generation of new mouse models whose MC deficiency is independent of restricted Kit gene expression (Müsch et al. 2008, Dudeck et al. 2011, Feyerabend etal. 2011) initiated a continuing controversy about the scientific significance of the Kit-dependent models (Katz \& Austen 2011, Brown et al. 2012) which were used for the investigation of MC biology in vivo during the last decades. The application of intravital microscopy to C57BL/6J-Mcpt5-Cre ROSA26-EYFP mice in which Mcpt5-producing MCs are detectable by the expression of EYFP (Scholten et al. 2008) represents a useful tool to further address the Kit-independent action of MCs and is of special interest for us.

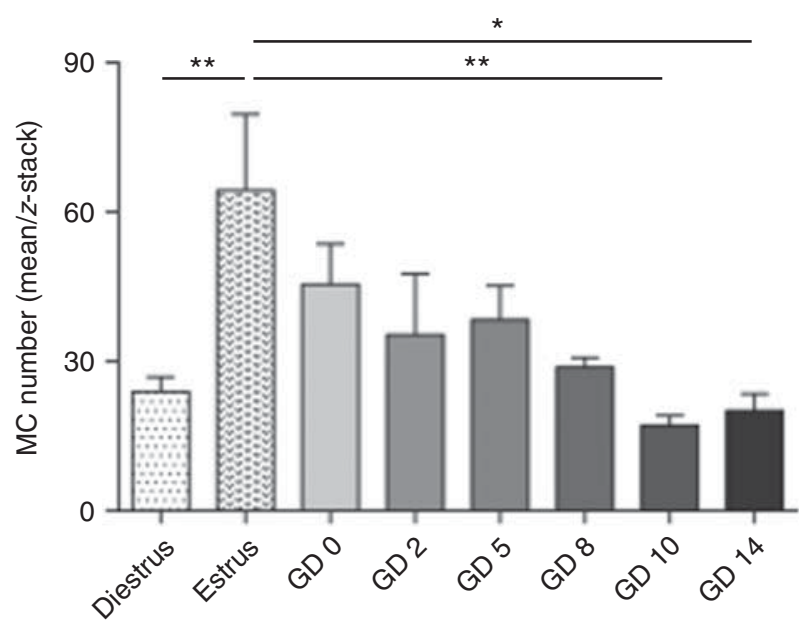

Figure 4 Intravital two-photon microscopy allows the quantification of uterine MCs (uMCs) in virgin and pregnant uteri of C57BL/6J-Mcpt5Cre ROSA26-EYFP mice. The number of MCs was determined in representative z-stacks of virgin or pregnant female C57BL/6J-Mcpt5Cre ROSA26-EYFP mice ( $n=5 /$ cycle in non-pregnant and $n=3 /$ stage in pregnant mice) using Zeiss LSM Image Browser software. Significantly higher numbers of uMCs were counted at estrus (receptive phase) in comparison to diestrus of the estrous cycle. At early pregnancy stages, the number of uMCs remained high and comparable to the values observed in estrus but declined as pregnancy advanced. Data were presented as mean \pm S.E.M. and differences were calculated using ANOVA test and Tukey's multiple comparison test post hoc, $* P<0.05$ and ${ }^{* *} P<0.005$. GD, gestational day. 
We adapted the technique introduced by Zenclussen et al. (2013) to the specific area of the uterus in which MCs are mainly distributed. Besides, we also investigated pregnant uteri that constituted a special challenge because of the increasing contractions as pregnancy advances. Herein, we report that imaging of the uterus was possible using a custom-made holder without any further manipulation of the tissue. MCs accumulated at the receptive phase of the female, namely in estrus when the uterine tissue is prepared for the nidation of the blastocyst. A similar distribution pattern could be observed live for DCs which additionally showed a clustering in estrus (Zenclussen et al. 2013) and are known to be crucial for the implantation process (Plaks et al. 2008). The accumulation of MCs at estrus could affect the physical as well as physiological contacts between the blastocyst and the uterine epithelium. In this study, a remarkably large number of uMCs at the mesometrial site of the virgin and pregnant uteri at all pregnancy stages were located in close proximity to blood vessels. To date, MC distribution pattern in uterus was exclusively referred to histological dying techniques applied to fixed tissue (Gaytan et al. 1991, Karaca et al. 2008). In this study, we demonstrate that the number of MCs in the uterus is much higher as it was suspected. We can further confirm their localization close to vessels; this may be beneficial for the release of MC-specific mediators into the vasculature. Moreover, the immediate vicinity of MCs located at the mesometrial region to the feto-placental unit suggests an interaction with other immune cells such as uterine NK cells, which are located within the mesometrial lymphoid aggregate of pregnancy (MLap) as well as within the decidua basalis (Croy et al. 2006). This together with the observation that uMCs are additionally located inter-implantational (Woidacki et al. 2013) suggests a significant role of UMCs and MC-specific mediators in reproductive processes such as implantation, placentation and later spiral artery remodeling. Recently published data point out that their absence is associated with abnormal implantation, aberrant placentation and inadequate spiral artery remodeling (Woidacki et al. 2013).

During the pre-implantation phase, the uterine tissue undergoes tremendous remodeling processes as a reaction to the invasion of fetal-derived trophoblasts (Rugh 1990). MCs are best known for their broad array of granule-stored mediators. The majority of these mediators are directly or indirectly involved in tissue remodeling and angiogenic processes including matrix metalloproteases, tryptases, chymases, TPA, TGF $\beta$, fibroblast growth factor and VEGF (Galli et al. 2005). During the implantation process when the trophoblast cells penetrate the uterine epithelium and decidualization starts, we found uMCs to be present and abundant at the mesometrial area of the uterus. In mice, the inner cell mass of the blastocysts is oriented mesometrially (Dey et al. 2004). The remarkably large amount of uMCs at the mesometrial site of the uterus before and during implantation strongly suggests that they exert a positive influence on the establishment of pregnancy. However, and in contrast to this concept, in 1996 a significant role for MCs in the process of implantation was excluded. This conclusion was based on a negligible amount of MCs found in uterine tissue (Salamonsen et al. 1996). Back then, the quantification of MCs was only possible ex vivo in fixed tissue by means of immunohistochemical techniques. The introduced visualization of numerous uMCs live and in vivo using intravital microscopy suggests that inadequate fixation procedures of the tissue could cause false results as suggested by El Sayed \& Dyson (1993). We did not perform confocal microscopy of fixed or unfixed tissue of C57BL/6J-Mcpt5-Cre ROSA26-EYFP mice to state whether this alternative method yields similar results as ours in in vivo observations. It is, however, evident from this study that MCs are present in significant numbers and at strategic sites at the fetal-maternal interface, which supports recent data on their important role for pregnancy establishment (Woidacki et al. 2013).

During the post-implantation phase, when placentation starts (day 8) (Rugh 1990) and spiral arteries appear within the decidua basalis (Burke et al. 2010), uMCs remained present in the uterus. Recently, we demonstrated that mice deficient in uMCs showed decreased placental surface areas and an inadequate remodeling of spiral arteries compared with WT C57BL/6J mice (Woidacki et al. 2013), whose uterine tissue is characterized by the presence of MCs at days 8 and 10 of pregnancy as shown in the present paper. Defective placental development as well as spiral artery formation cause fetal growth retardation (Ashkar et al. 2000, Song et al. 2002, Watson \& Cross 2005) as both structures are crucial for maternal and fetal blood exchange, and therefore, for supplying the fetus with oxygen and nutrients. Even at day 14 of pregnancy when the fetus but not the placenta needs to grow (Suzuki et al. 1997), the amount of uMCs is still considerable although significantly lower when compared with that at estrus. These observations are indicative of the involvement of uMCs in pregnancy establishment rather than its maintenance. This may be due to the secretion of soluble factors contained in their granules. One important mediator was found to be Gal1. MCs deficient in Lgals1 were not efficient in restoring placental growth as MCs from WT animals did when transferred into Kit-deficient mice (Woidacki et al. 2013). It is, however, possible that other $\mathrm{UMC}$ mediators are involved in this late process, too. Moreover, based on our data (Woidacki et al. 2013), we speculated that the mucosal MC phenotype that is also present in uterine tissue plays a role in mediating pre-, peri- and post-implantation events. Thus, the unique uterine micromilieu during pregnancy might influence the distribution/expression balance between both the MC phenotypes. The detection of uMCs that undergo a 
transdifferentiation process (Woidacki et al. 2013) might support this assumption.

Although the detected EYFP-positive cells represent connective tissue-type MCs based on the expression of the serine protease Mcpt-5 (Dudeck et al. 2011) and uMCs display a unique population composed of mucosal, connective tissue-type and a third transdifferentiation cell-type MCs (Spicer 1960, Woidacki et al. 2013), C57BL/6J-Mcpt5-Cre ROSA26-EYFP mice are a valuable and reliable model for studying uMC behavior live and in vivo. To the best of our knowledge, except one alternative model that is based on a cross of a ROSA26tdRFP reporter strain (Luche et al. 2007) with the Mcpt5Cre transgenic mouse strain (C57BL/6J-Mcpt5-Cre Rosa26-tdRFP) (Roers A 2012, personal communication), no other MC-specific reporter strains are available.

During the imaging process, uterine contractions, which would have negatively influenced the quality of pictures and movies, were reduced to a minimum by applying caffeine-soaked cotton rolls that ensured a permanent drenching of the uterus. Former problems of intravital technology concerning the amenability of the organ could be resolved by performing handling modifications of the animal and uterus respectively. According to the applied imaging procedures and a custom-made microscope stage, intravital images of placenta (Zenclussen et al. 2012) and uterus (Zenclussen et al. 2013) emerged as an adequate appliance for the basic fixation of the uterus for analyzing uMCs. A selfmade fixator composed of tear-resistant aluminum foil served as a fine fixation for the uterus. The addition of low-melting agarose allowed a constant and long-lasting moistening of the focal area.

The application of two-photon microscopy to C57BL/6J-Mcpt5-Cre ROSA26-EYFP mice represents a novel and exciting approach to track $M C$ behavior in vivo. It could be a powerful tool to bring to light the so-far controversial discussed influence of MCs on reproductive processes and pregnancy. We do hope that the introduced visualization of uMCs might help in the future for addressing elementary questions regarding MCs and their participation in physiological and pathological processes.

\section{Declaration of interest}

The authors declare that there is no conflict of interest that could be perceived as prejudicing the impartiality of the research reported.

\section{Funding}

This work was supported by grants from the German Research Council (DFG) to A C Zenclussen (DFG ZE 526/6-1). A C Zenclussen, K Woidacki, F Schmerse and A Roers are members of the SPP 1394 of the DFG. A C Zenclussen and A Roers are members of the COST Action 1007.

\section{Acknowledgements}

We thank Markus Scharm for his excellent technical assistance, Anne Dudeck for providing the C56BL/6J-Mcpt5-Cre ROSA26EYFP mice and Lars Philipsen for valuable assistance with the two-photon microscope technique.

\section{References}

Anand P, Singh B, Jaggi AS \& Singh N 2012 Mast cells: an expanding pathophysiological role from allergy to other disorders. NaunynSchmiedeberg's Archives of Pharmacology 385 657-670. (doi:10.1007/ s00210-012-0757-8)

Ashkar AA, Di Santo JP \& Croy BA 2000 Interferon $\gamma$ contributes to initiation of uterine vascular modification, decidual integrity, and uterine natural killer cell maturation during normal murine pregnancy. Journal of Experimental Medicine 192 259-270. (doi:10.1084/jem.192.2.259)

Bischoff SC 2009 Physiological and pathophysiological functions of intestinal mast cells. Seminars in Immunopathology 31 185-205. (doi:10.1007/ s00281-009-0165-4)

Bosquiazzo VL, Ramos JG, Varayoud J, Muñoz-de-Toro M \& Luque EH 2007 Mast cell degranulation in rat uterine cervix during pregnancy correlates with expression of vascular endothelial growth factor mRNA and angiogenesis. Reproduction 133 1045-1055. (doi:10.1530/REP-060168)

Brown MA, Hatfield JK, Walker ME \& Sayed BA 2012 A game of kit and mouse: the Kit is still in the bag. Immunity 36 891-892. (doi:10.1016/ j.immuni.2012.05.004)

Burke SD, Barrette VF, Gravel J, Carter AL, Hatta K, Zhang J, Chen Z, Leno-Durán E, Bianco J, Leonard S et al. 2010 Uterine NK cells, spiral artery modification and the regulation of blood pressure during mouse pregnancy. American Journal of Reproductive Immunology 63 472-481. (doi:10.1111/j.1600-0897.2010.00818.x)

Croy BA, van den Heuvel MJ, Borzychowski AM \& Tayade C 2006 Uterine natural killer cells: a specialized differentiation regulated by ovarian hormones. Immunological Reviews 214 161-185. (doi:10.1111/j.1600065X.2006.00447.x)

De Moraes LV, Tadokoro CE, Gómez-Conde I, Olivieri DN \& PenhaGonçalves C 2013 Intravital placenta imaging reveals microcirculatory dynamics impact on sequestration and phagocytosis of Plasmodiuminfected erythrocytes. PLoS Pathogens 9 e1003154. (doi:10.1371/ journal.ppat.1003154)

Dey SK, Lim H, Das SK, Reese J, Paria BC, Daikoku T \& Wang H 2004 Molecular cues to implantation. Endocrine Reviews 25 341-373. (doi:10.1210/er.2003-0020)

Dudeck A, Dudeck J, Scholten J, Petzold A, Surianarayanan S, Köhler A, Peschke K, Vöhringer D, Waskow C, Krieg T et al. 2011 Mast cells are key promoters of contact allergy that mediate the adjuvant effects of haptens. Immunity 34 973-984. (doi:10.1016/j.immuni.2011.03.028)

El Sayed SO \& Dyson M 1993 Histochemical heterogeneity of mast cells in rat dermis. Biotechnic \& Histochemistry 68 326-332. (doi:10.3109/ 10520299309105638)

Feyerabend TB, Weiser A, Tietz A, Stassen M, Harris N, Kopf M, Radermacher P, Möller P, Benoist C, Mathis D et al. 2011 Cre-mediated cell ablation contests mast cell contribution in models of antibodyand $\mathrm{T}$ cell-mediated autoimmunity. Immunity 35 832-844. (doi:10.1016/ j.immuni.2011.09.015)

Galli SJ \& Tsai M 2012 IgE and mast cells in allergic disease. Nature Medicine 18 693-704. (doi:10.1038/nm.2755)

Galli SJ, Nakae S \& Tsai M 2005 Mast cells in the development of adaptive immune responses. Nature Immunology 6 135-142. (doi:10.1038/ ni1158)

Gaytan F, Aceitero J, Bellido C, Sánchez-Criado JE \& Aguilar E 1991 Estrous cycle-related changes in mast cell numbers in several ovarian compartments in the rat. Biology of Reproduction 45 27-33. (doi:10. 1095/biolreprod45.1.27)

Holinka CF, Tseng YC \& Finch CE 1979 Reproductive aging in C57BL/6) mice: plasma progesterone, viable embryos and resorption frequency throughout pregnancy. Biology of Reproduction 20 1201-1211. (doi:10. 1095/biolreprod20.5.1201) 
Jensen F, Woudwyk M, Teles A, Woidacki K, Taran F, Costa S, Malfertheiner SF \& Zenclussen AC 2010 Estradiol and progesterone regulate the migration of mast cells from the periphery to the uterus and induce their maturation and degranulation. PLOS ONE 5 e14409. (doi:10.1371/journal.pone.0014409)

Karaca T, Arikan S, Kalender H \& Yoruk M 2008 Distribution and heterogeneity of mast cells in female reproductive tract and ovary on different days of the oestrus cycle in Angora goats. Reproduction in Domestic Animals 43 451-456. (doi:10.1111/j.1439-0531.2007.00934.x)

Katz HR \& Austen KF 2011 Mast cell deficiency, a game of kit and mouse. Immunity 35 668-670. (doi:10.1016/j.immuni.2011.11.004)

Luche H, Weber O, Nageswara Rao T, Blum C \& Fehling HJ 2007 Faithful activation of an extra-bright red fluorescent protein in "knock-in" Crereporter mice ideally suited for lineage tracing studies. European Journal of Immunology 37 43-53. (doi:10.1002/eji.200636745)

Lützelschwab C, Huang MR, Kullberg MC, Aveskogh M \& Hellman L 1998 Characterization of mouse mast cell protease-8, the first member of a novel subfamily of mouse mast cell serine proteases, distinct from both the classical chymases and tryptases. European Journal of Immunology 28 1022-1033. (doi:10.1002/(SICl)1521-4141(199803)28:03<1022:: AID-IMMU1022 > 3.0.CO;2-1)

Menzies FM, Higgins CA, Shepherd MC, Nibbs RJ \& Nelson SM 2012 Mast cells reside in myometrium and cervix, but are dispensable in mice for successful pregnancy and labor. Immunology and Cell Biology 90 321-329. (doi:10.1038/icb.2011.40)

Metcalfe DD, Baram D \& Mekori YA 1997 Mast cells. Physiological Reviews 77 1033-1079.

Michaloudi HC \& Papadopoulos GC 1999 Mast cells in the sheep, hedgehog and rat forebrain. Journal of Anatomy 195 577-586. (doi:10. 1046/j.1469-7580.1999.19540577.x)

Müsch W, Wege AK, Männel DN \& Hehlgans T 2008 Generation and characterization of $\alpha$-chymase-Cre transgenic mice. Genesis $\mathbf{4 6}$ 163-166. (doi:10.1002/dvg.20378)

Olivieri DN \& Tadokoro CE 2013 Techniques for visualization of cell-cell contact at the fetal-maternal interface. American Journal of Reproductive Immunology 69 419-426. (doi:10.1111/aji.12070)

Paoli J, Smedh M \& Ericson MB 2009 Multiphoton laser scanning microscopy - a novel diagnostic method for superficial skin cancers. Seminars in Cutaneous Medicine and Surgery 28 190-195. (doi:10.1016/ j.sder.2009.06.007)

Parkening TA, Lau IF, Saksena SK \& Chang MC 1978 Circulating plasma levels of pregnenolone, progesterone, estrogen, luteinizing hormone, and follicle stimulating hormone in young and aged C57BL/6 mice during various stages of pregnancy. Journal of Gerontology 33 191-196. (doi:10.1093/geronj/33.2.191)

Plaks V, Birnberg T, Berkutzki T, Sela S, BenYashar A, Kalchenko V, Mor G, Keshet E, Dekel N, Neeman M et al. 2008 Uterine DCs are crucial for decidua formation during embryo implantation in mice. Journal of Clinical Investigation 118 3954-3965. (doi:10.1172/JCl36682)

Rugh R 1990 The Mouse - Its Reproduction and Development. Oxford University Press.

Salamonsen LA, Jeziorska M, Newlands GF, Dey SK \& Woolley DE 1996 Evidence against a significant role for mast cells in blastocyst implantation in the rat and mouse. Reproduction, Fertility and Development 8 1157-1164. (doi:10.1071/RD9961157)

Scholten J, Hartmann K, Gerbaulet A, Krieg T, Müller W, Testa G \& Roers A 2008 Mast cell-specific Cre/loxP-mediated recombination in vivo. Transgenic Research 17 307-315. (doi:10.1007/s11248-007-9153-4)

Schwartz LB \& Huff TF 1998 Biology of mast cells. In Allergy: Principles and Practice, vol 1, pp 261-276. Eds EJ Middleton, CE Reed, EF Ellis, JW Yunginger, NFJ Adkinson \& WW Busse. St Louis, MO: Mosby-Year Book.
Song H, Lim H, Paria BC, Matsumoto H, Swift LL, Morrow J, Bonventre JV \& Dey SK 2002 Cytosolic phospholipase A2 $\alpha$ is crucial for 'on-time' embryo implantation that directs subsequent development. Development 129 2879-2889.

Spicer SS 1960 A correlative study of the histochemical properties of rodent acid mucopolysaccharides. Journal of Histochemistry \& Cytochemistry $\mathbf{8}$ 18-35. (doi:10.1177/8.1.18)

Srinivas S, Watanabe T, Lin CS, William CM, Tanabe Y, Jessell TM \& Costantini F 2001 Cre reporter strains produced by targeted insertion of EYFP and ECFP into the ROSA26 locus. BMC Developmental Biology 4 e462.

St John AL \& Abraham SN 2013 Innate immunity and its regulation by mast cells. Journal of Immunology 190 4458-4463. (doi:10.4049/jimmunol. 1203420)

Suzuki K, Kobayashi M, Kobayashi K, Shiraishi Y, Goto S \& Hoshino T 1997 Structural and functional change of blood vessel labyrinth in maturing placenta of mice. Placenta 18 155-164. (doi:10.1016/S0143-4004(05) 80169-9)

Talbert GB 1971 Effect of maternal age on postimplantation reproductive failure in mice. Journal of Reproduction and Fertility 24 449-452. (doi:10.1530/jrf.0.0240449)

Teles A, Schumacher A, Kühnle MC, Linzke N, Thuere C, Reichardt $P$, Tadokoro CE, Hämmerling GJ \& Zenclussen AC 2013 Control of uterine microenvironment by Foxp3(+) cells facilitates embryo implantation. Frontiers in Immunology 20 158. (doi:10.3389/fimmu.2013.00158)

Theoharides TC, Alysandratos KD, Angelidou A, Delivanis DA, Sismanopoulos N, Zhang B, Asadi S, Vasiadi M, Weng Z, Miniati A et al. 2010 Mast cells and inflammation. Biochimica et Biophysica Acta 1822 21-33. (doi:10.1016/j.bbadis.2010.12.014)

Varayoud J, Ramos JG, Bosquiazzo VL, Muñoz-de-Toro M \& Luque EH 2004 Mast cells degranulation affects angiogenesis in the rat uterine cervix during pregnancy. Reproduction 127 379-387. (doi:10.1530/rep. 1.00018)

Wang H \& Dey SK 2006 Roadmap to embryo implantation: clues from mouse models. Nature Reviews. Genetics 7 185-199. (doi:10.1038/ nrg1808)

Watson ED \& Cross JC 2005 Development of structures and transport functions in the mouse placenta. Physiology 20 180-193. (doi:10.1152/ physiol.00001.2005)

Williams RM, Zipfel WR \& Webb WW 2005 Interpreting second-harmonic generation images of collagen I fibrils. Biophysical Journal $\mathbf{8 8}$ 1377-1386. (doi:10.1529/biophysj.104.047308)

Woidacki K, Popovic M, Metz M, Schumacher A, Linzke N, Teles A, Poirier F, Fest S, Jensen F, Rabinovich GA et al. 2013 Mast cells rescue implantation defects caused by c-kit deficiency. Cell Death \& Disease 4 e462. (doi:10.1038/cddis.2012.214)

Zenclussen AC, Olivieri DN, Dustin ML \& Tadokoro CE 2012 In vivo multiphoton microscopy technique to reveal the physiology of the mouse placenta. American Journal of Reproductive Immunology 68 271-278. (doi:10.1111/j.1600-0897.2012.01161.x)

Zenclussen AC, Olivieri DN, Dustin ML \& Tadokoro CE 2013 In vivo multiphoton microscopy technique to reveal the physiology of the mouse uterus. American Journal of Reproductive Immunology 69 281-289. (doi:10.1111/aji.12066)

Zipfel WR, Williams RM \& Webb WW 2003 Nonlinear magic: multiphoton microscopy in the biosciences. Nature Biotechnology 21 1369-1377. (doi:10.1038/nbt899)

Received 6 November 2013

First decision 19 December 2013

Revised manuscript received 15 January 2014

Accepted 10 February 2014 